\title{
Post-Tracheostomy Care: Bundle Up for Success!
}

Tracheostomy tube placement is a commonly performed procedure in the ICU indicated as an alternative method to establish an artificial airway. The decision to insert a tracheostomy tube relies on a few indications: (1) patients who require a bypass for upper airway obstruction or compromise (eg, head-neck cancer); (2) patients who have severe and prolonged depressed levels of consciousness; (3) patients with neuromuscular disease and respiratory muscle weakness who fail to tolerate noninvasive methods to ventilate and clear secretions; and (4) complex medical and surgical critically ill patients who fail to wean off mechanical ventilation. ${ }^{1}$

There are several advantages of a tracheostomy tube, including liberation of the oropharynx, secure and easy airway manipulation, and less need for deep sedation. These advantages could potentially help restore phonation, oral intake, and participation in rehabilitation. ${ }^{2}$

Tracheostomy tubes may be permanent or temporary, depending on their indications. For temporary tubes, the duration from insertion to decannulation is variable, but it is accepted that this process can take weeks or months, depending on the patient's potential to recover from the initial insult that led to medical ventilation. ${ }^{3}$ Regardless, a patient's quality of life can be significantly impacted throughout the process of liberation from mechanical ventilation and decannulation by attending to their need for phonation, deglutition, and secretion management.

This important aspect of multidisciplinary and coordinated care of tracheostomized patients was addressed by Mah in this issue of Respiratory CARE. ${ }^{4}$ In this singlecenter study, the investigators compared 3 historical cohorts that reflect the change in practice from usual care, to an intensivist-led tracheostomy management service, to a post-tracheostomy care bundle. The latter consists of an electronic postoperative order set with automated respiratory therapist and speech language pathologist consultations, a tracheostomized patient tracking and following system, a respiratory therapist-driven decannulation pro-

Dr Divo has disclosed no conflicts of interest.

Correspondence: Miguel J Divo MD, Pulmonary and Critical Care Division, Brigham and Women's Hospital, 75 Francis Street, Boston, MA 02115. E-mail: mdivo@partners.org.

DOI: $10.4187 /$ respcare 05410 tocol, and a formal rounding process to individualize tracheostomy care. Despite it being a single-center study, they included 393 subjects and demonstrated that use of a post-tracheostomy care bundle improved the decannulation rate from 8 to $26 \%$ and initiation of oral diet from 16 to $35 \%$ as compared with their historical baseline usual care cohort. The impact of these management changes on patients and caregivers is not trivial.

See the Original Study on Page 137

The key point of their intervention is the application of a common sense approach to tracheostomy care. This approach does not require the use of new devices or complex interventions, but rather organizes or bundles common clinical practices used in many centers. Bundling and checklists are important components in the care of this group of complex and vulnerable patients. They allow the provision of evidence-based care to everyone, every time, in a setting of complex care with many moving parts. It is easy to oversee, delay, or ignore certain aspects of critical care, particularly when those tasks are performed by different teams (respiratory care, speech language pathologists, nursing, etc) and their members differ from shift to shift over the course of hospital stay (median of $36 \mathrm{~d}$ for patient with a tracheostomy!). ${ }^{4}$

Although the results of this study are encouraging, they should be interpreted with some caution. There were significant differences in the baseline characteristics of the reference group (usual care) that may have influenced the study's findings. The usual care group of 61 subjects had higher APACHE (Acute Physiology and Chronic Health Evaluation) and SOFA (Sequential Organ Failure Assessment) scores. These are markers of disease severity and prognosis. In addition, there were fewer trauma subjects in this group (20\% compared with $36 \%$ in the bundle group). This is significant in that trauma patients tend to be younger and have fewer comorbid conditions, which favors successful decannulation.

How should we interpret the findings of this study? Taken together, the results reported by Mah et $\mathrm{al},{ }^{4}$ as well as those from the meta-analysis by Speed et al, ${ }^{5}$ show that bundling multiple facets of tracheostomy care makes sense and is a crucial step to improve the outcomes of tracheostomy patients. The importance of the Mah et al study 
can be seen when compared with 2 previous care strategies, in which similar interventions were applied without the structure of the bundle. The synergistic and coordinated effect of this multifaceted approach have a greater impact than when treatment is only based on its individual components.

Future studies should attempt to replicate these results to establish external validity. In addition, it is essential to further examine how to improve the individual components of tracheostomy care in the context of bundling multiple interventions. This research should include appropriate sizing and fitting of tubes, determining the best decannulation protocol, providing objective evidence on how to re-initiate phonation and deglutition, and how to extend those interventions when patients are transferred to stand-alone institutions, such as long-term acute care hospitals or step down units.

Miguel J Divo MD

Pulmonary and Critical Care Division Brigham and Women's Hospital
Boston, Massachusetts

Respiratory Services

Spaulding Rehabilitation Hospital

Cambridge, Massachusetts

Harvard Medical School

Boston, Massachusetts

\section{REFERENCES}

1. Hess DR. Tracheostomy tubes and related appliances. Respir Care 2005;50(4):497-510.

2. Epstein SK. Anatomy and physiology of tracheostomy. Respir Care 2005;50(4):476-482.

3. Frutos-Vivar F, Esteban A, Apezteguía C, Anzueto A, Nightingale P, González M, et al. Outcome of mechanically ventilated patients who require a tracheostomy. Crit Care Med 2005;33(2):290-298.

4. Mah JW, Staff I, Fisher S, and Butler KL. Improving decannulation and swallowing function: a comprehensive, multidisciplinary approach to post-tracheostomy care. Respir Care 2017;62(2):137-143.

5. Speed L, Harding KE. Tracheostomy teams reduce total tracheostomy time and increase speaking valve use: a systematic review and metaanalysis. J Crit Care 2013;28:216.e1-216.e10. 